\title{
ENABLING MOLECULAR COMMUNICATION THROUGH CHIRALITY OF ENANTIOMERS
}

\author{
Valeria Loscrí ${ }^{1}$ and Anna Maria Vegni ${ }^{2}$ \\ ${ }^{1}$ Inria Lille - Nord Europe, Lille, France, ${ }^{2}$ Roma Tre University, Rome, Italy \\ NOTE: Corresponding author: Valeria Loscrí, valeria.loscri@inria.fr
}

\begin{abstract}
With the advancement of nanotechnology, there has been fervid research activity on new communication paradigms suitable for new challenging contexts, such as biological systems. Among different approaches, the most considered has been artificial Molecular Communication, where entities such as synthetic molecules, enzymes, hormones, bacteria etc. are functionalized in order to implement information exchange with the surrounding system and with other entities. In this context, it is interesting to analyze specific features that could be exploited for effective communication paradigms. In this paper, we focus on chiral molecules (a.k.a. enantiomers) as novel enablers for a molecular communication paradigm. Chirality is an interesting and appealing feature existing in nature and that can be replicated with strong emphasis in new types of materials, such as metasurfaces and metamaterials. A deep knowledge of chirality features and how chiral molecules interact with each other or with achiral molecules provides insights into designing a new molecular communication technique suitable for biological environments. In this contribution, we will highlight the main applications of chiral molecules and we will present chiral features as the viable way for realizing a nanocommunication system.
\end{abstract}

Keywords - Chiral molecules, chirality transfer, enantiomers, molecular communications, optical activity

\section{INTRODUCTION}

A Molecular Communication (MC) paradigm consists of using molecules to encode, transmit and receive information. It has recently received a lot of attention by the research community since it is considered as the viable alternative of electromagnetic (EM) communications, thanks to the specific features of biocompatibility. MC is mostly inspired by existing communication mechanisms occurring between biological entities and is developed by considering small molecules, peptides, lipids, as well as bacteria, viruses, pheromones and so on [1].

An MC paradigm is based on the transmission and reception of information encoded into molecules (i.e., messenger molecules) $[2,3]$. These entities freely propagate in the medium by connecting a transmitter with a receiver nanomachine. Typical molecular communication systems are based on the free diffusion process of molecules, such as calcium signaling, microtubules, pheromone signaling, and bacterium-based communications [4]. Different biological entities allow reaching different communication ranges and performance. For instance, the use of pheromones (i.e., molecules of chemical compounds released by plants, insects, and other animals) triggers specific behaviors among the receptor members and reaches long-range communications i.e., approximately one meter. On the other side, both flagellated bacteria and catalytic nanomotors are able to carry DNA messages and allow short-range communications. The use of DNA as information messages allows achieving an information rate that is relatively high (i.e., up to several kilobits per second). In contrast, the propagation of information by means of guided bacteria or catalytic nanomotors is relatively very slow (i.e., a few millimeters per hour).

A special type of molecules that is expected to be very promising in the field of molecular communications is the chiral molecule. Chiral molecules show the chirality effect i.e., a physical phenomenon that pervades the universe. The term chirality was introduced in 1884 and refers to objects that are not equivalent to their mirror images, and the two images are not superposed to each other. A typical example of such a geometrically chiral object is the human hand, so that the left and the right hands are mirror images of each other, but it is impossible to superpose them.

Chiral molecules, a.k.a. enantiomers, can show their different handedness in many ways, including the way they affect human beings. For instance, one enantiomeric form of a compound called limonene is primarily responsible for the odor of oranges, while the other enantiomer, for the odor of lemons. Molecules of the amino acids of which our proteins are built have the property of being non-superposable on their mirror image. In contrast, objects (and molecules) that are superposable on their images are achiral. The chirality of molecules can be demonstrated with relatively simple compounds. For instance, consider a 2-butanol molecule i.e., an organic compound with formula $\mathrm{CH}_{3} \mathrm{CH}(\mathrm{OH}) \mathrm{CH}_{2} \mathrm{CH}_{3}$. The structure of 2-butanol is chiral, that means that there are actually two different 2-butanols and they are enantiomers. Another example is the amino acid alanine, which is in the form of left-handed and right-handed enantiomers i.e., (S)-alanine and (R)-alanine, respectively. Fig. 1 depicts 
the two enantiomers, whose mirror images are not equivalent and non-superposable on each other. Another example of enantiomers are the molecules of natural sugars, almost all classified as being right handed, including the sugar that occurs in DNA. Also DNA is a chiral structure, since its two helixes are not superposable to each other, as well depicted in Fig. 1 (b).

In this paper we address how to exploit chiral molecules as messenger molecules for molecular communications. The use of similar molecules i.e., isomers, as enablers of molecular communications has already been investigated in [5], where Kim and Chae proposed three novel modulation techniques, i.e., concentration-based, moleculartype-based, and molecular-ratio-based. However, in [5] it did not emerge the main features of such special molecules and how it is possible to exploit them for molecular communications by means of their inner features. For example, one of the main features of chiral molecules is their behavior towards plane-polarized light. When a beam of plane-polarized light passes through an enantiomer, the plane of polarization rotates. Also, separate enantiomers rotate the plane of plane-polarized light at equal amounts but in opposite directions. Then, separate enantiomers are optically active compounds.

In this paper, we focus on the features of chiral molecules such as (i) the rotation of the polarization plane of the impinging EM wave and (ii) the chirality transfer effect, in order to model a chiral channel comprised of enantiomers that forward data information via a multi-hop protocol. Specifically, in our vision, data information is represented by exploiting the chirality phenomenon with a chiral molecule emitting a rotated EM wave when a light input impinges on an initial transmitter node after a steady state is achieved. Dissemination of data information inside a chiral medium occurs through the chirality transfer mechanism that considers the non-covalent bonds between a chiral and an achiral molecule. Chirality is exploited to encode the information in the chiral molecules, and it is decoded at the receiver as a 1 bit when an EM wave (i.e., an optical signal) is applied. When no EM wave is applied, the information is decoded as a bit 0 .

This paper is organized as follows. Section 2 introduces the concept of chirality effect and describes the main enantiomers that can be found in nature, specifically in the biological context. The features of chiral molecules are then presented in Subsection 2.1. In Section 3 we characterize the chiral transfer effect from a chiral molecule to an achiral molecule. In Section 4, we define a chiral medium as a channel for molecular communications. Data information is encoded in the chiral molecules that transport the chirality effect, which can be forwarded hop-by-hop in the overall system. Finally, conclusions are drawn at the end of this paper.

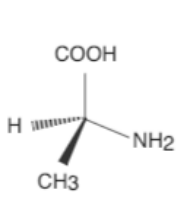

(S)-alanine
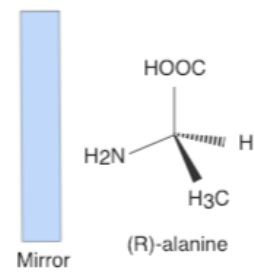

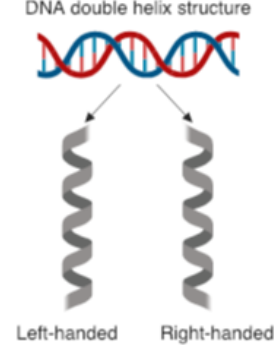

(b)
Fig. 1 - Examples of chiral molecules in case of (a) alanine amino-acid, and (b) DNA chains. Enantiomers are non-superposable mirror images to each other.

\section{CHIRAL MOLECULES}

A chiral molecule shows the chirality effect that makes the molecule not equivalent to its mirror image. The chiral molecule and its mirror image are enantiomers, and the relationship between the chiral molecule and its mirror image is defined as enantiomeric. The word chiral comes from the Greek, and means "hand". Indeed, a classic example of chiral objects are the hands, since the mirror image of the left hand is exactly the right hand. However, the left hand is not superposable on the right hand.

From the etymology of chiral, chiral objects are said to possess "handedness". Although both mirror image forms are theoretically possible, such as those for the amino acid alanine, they have evolved in a way that amino acids are mainly of the mirror image said to be "left-handed" (see Fig. 1 (a)). The reason that most amino acids are of the left-handed form is not known, however.

Chirality is an important phenomenon in the universe. Several plants show chirality, by winding around supporting structures. The human body is structurally chiral and it is not clear why, but most people are right-handed. Usually, only one form of chiral occurs in a given species. Just as an example, the molecules of white sugars are right handed. Enantiomers of a chiral molecule have identical physico-chemical properties, and also the same electrochemical behavior. The enantioselective electrochemistry represents the ability of discriminating enantiomers of chiral molecules (i.e., electroanalysis), or to selectively activate or achieve a given enantiomer of a chiral molecule (i.e., electrosynthesis) and is an issue particularly important in the biological and pharmaceutical fields [6].

\subsection{Features of chiral molecules}

The specific rotation is a property of a chiral molecule. It is defined as the change in orientation of monochromatic plane-polarized light, per unit distance-concentration product, as the light passes through a sample of a compound in solution. Fig. 2 describes the property of rotation of the polarization plane of an EM field that impinges on a chiral channel. At the output of the channel, the polarization plane has been rotated. Specifically, chiral molecules can rotate the plane of polarization of an 


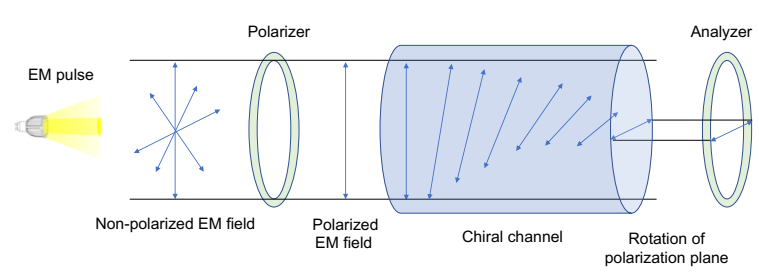

Fig. 2 - Property of rotation of the polarization plane of an EM pulse impinging a chiral channel.

EM field both clockwise and counterclockwise. If the rotation is clockwise, chiral molecules are said to be dextrorotary, and correspond to positive rotation values, while chiral molecules rotating the plane of polarization counterclockwise are said to be levorotary, and correspond with negative values of the specific rotation.

Another important feature of chiral molecules is the chirality transfer that occurs when a chiral molecule encounters an achiral molecule. In such a scenario, the chirality effect is extended over the whole molecular system i.e., it propagates from a chiral to an achiral molecule, which becomes chiral. The induction of the chirality in the achiral components is of utmost importance. In order to induce the chirality of the achiral components, the interaction between the chiral molecules and the achiral molecules plays a very important role.

The induced chirality generally refers to those chiral supramolecular systems where chirality is induced in an achiral guest molecule as a result of asymmetric information transfer from a chiral host e.g., a chiral molecule or a chiral nanostructure. In order to produce the induced chirality, it is necessary for the achiral molecule to have a strong interaction with the chiral host through a noncovalent bond. A typical example of induced chirality is the encapsulation of a chromophore into the cavity of cyclodextrin [7]. Finally, a very important aspect related to chirality is the chiral communication that is a common phenomenon occurring in many biological processes [8], strictly tied with the chirality transfer property.

In this paper, we will exploit the chirality transfer effect by the means of diffusion of chiral molecules which come into contact with achiral molecules in a biological solution.

\section{CHIRALITY TRANSFER EFFECT}

As already introduced in Subsection 2.1, the chirality transfer effect is observed between organic and inorganic molecular structures. The modeling of macroscopic chi- rality emerged from the chiral molecular elements is a challenge for theory, computations, and experiments. $\mathrm{Nu}$ - merous experimental results demonstrated the transfer of chirality among different length scales ranging from di- mensions of the elementary particles to the macro-scale (i.e., the length of the axon). In particular, it was shown that the chirality at the molecular scale (i.e., amino acids, proteins, and polysaccharides) could be transferred to the macroscopic and macro level (i.e., neurofilaments and in-organic crystals).
In general, chirality transfer occurs through chemical bonds, but recently it has been observed that chiral biomolecules may impart some of their optical properties to a spatially separated achiral dye [9]. Knof and von Zelewsky [10] have characterized the chiral transfer as through the use of organic ligands chiral information can be transferred.

In the context of chirality transfer it is important to highlight chiroptical properties. Among the most important properties are the chiral luminescent lanthanide complexes used as probes for the characterization of chiral environments $[11,12,13]$ or as chiral luminescent complexes $[14,15,16,17]$. Lanthanides are ideal candi- dates as luminescent probes, based on specific features such as their long lifetimes and large Stokes shifts. CP luminescence has great potential to investigate the configurational, as well as conformational changes, in biological systems in solution, since it combines the gen- eral sensitivity of luminescence measurements and the high specificity of the signal for the chiral environment. Furthermore, using very simple chiral ligands and lanthanide ions, chiral nanoballs were obtained where the array of lanthanide ions are arranged as in the ferritin biological molecule $[18,19]$. With very simple chiral ligands used as synthons (i.e., a synthon is a component of a molecule to be synthesized, playing an active role in synthesis) in coordination chemistry, it is possible to obtain sophisticated chiral assemblies which mimic biological systems. These considerations are encouraging in the development of "artificial" molecular communication systems.

Chirality is also important in molecular switches [20,21]. A switch is a molecule that can reversibly interconvert be- tween two stable states upon an external stimulus. In [22] Dai et al. described a chiroptical switch based on pho- tochromes that exhibit two different states with signifi- cantly different optical rotations. Finally, chiral transfer phenomena can be used for sensing chirality of a wide range of chiral molecules, as well as for developing novel chiroptical devices and chiral materials. The wider application of chiral sensing continues to be hampered by the involved chiral signals being inherently weak. To avoid this issue, plasmonic and dielectric nanostructures have recently been shown to offer a viable route for enhancing weak circular dichroism (CD) effects. Re- cently, in [23] Mohammadi et al. presented an analytical study of the problem of substrate CD spectroscopy for an arbitrary nanophotonic substrate (either, chiral or achi- ral, plasmonic or dielectric) and clarify the interplay be- tween key affecting parameters, such as the thickness and chirality of the substrate, as well as the near-field optical chirality enhancement. From the telecommunications point of view, chirality transfer can be exploited by the means of a diffusion process of the chiral molecules. When the "chirality effect" is transferred to an achiral molecule and an optical signal is applied, it will be able to 


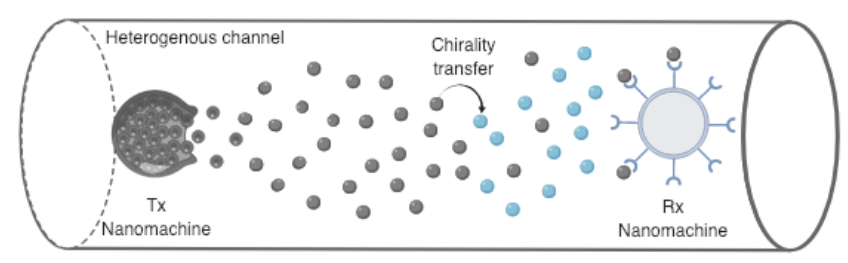

Fig. 3 - Chirality transfer property of chiral molecules (black circles) towards achiral molecules (light blue molecules).

show an optical activity, and become a chiral molecule. As a result, the chirality transfer works as point-to-point data forwarding among heterogeneous molecules (i.e., from chiral to achiral molecules).

Specifically, when a chiral molecule encounters an achiral component, it forms a non-covalent bond and the chirality effect is transferred into the achiral molecule, which becomes chiral. Finally, the chirality transfer propagates in the whole system.

Fig. 3 depicts the chirality transfer feature of chiral molecules in a heterogeneous channel (i.e., comprised of both chiral and achiral molecules). In this scenario, the chiral molecules are used as messenger molecules released by a transmitter nanomachine (e.g., a eukaryotic cell) through the medium via diffusion (i.e., Brownian motion). They are used as messengers, since they allow the transmission of a light signal applied to a transmitter molecule and transferred from a molecule to the neighbors through the chiroptical properties when the system will reach a steady state. The motion is basically driven by diffusion, meaning that the particles move from areas of higher concentration to areas of lower concentration, and the displacement of messenger molecules follows a normal distribution with zero mean.

The overall chiral molecule concentration flux is given by the sum of the $N$ chiral molecules concentration gradients, with $N$ as the number of apertures of the Tx nanomachine. The flux of chiral molecule concentration depends on both time and position through the Fick's first law i.e.,

$$
J(x, t)=-D \sum_{i=1}^{N} \nabla C_{i, C M}(x, t),
$$

where $\nabla$ is an operator used in vector calculus as a vector differential operator, $C_{i, C M}\left[\mathrm{~mol} / \mathrm{cm}^{3}\right]$ is the $i$-th chiral molecule concentration with $i=\{1,2, \ldots, N\}$, and $D$ $\left[\mathrm{cm}^{2} / \mathrm{s}\right]$ is the diffusion coefficient, assumed as a constant value for a given fluidic medium as:

$$
D=\frac{k_{B} T}{3 \pi \eta d}
$$

where $k_{B}$ is the Boltzmann constant equal to $1.38 \times$ $10^{-23}[\mathrm{~J} / K], T$ is the temperature $[K], \eta$ is the viscosity of the liquid $[\mathrm{mPa} \cdot \mathrm{s}]$, and $d$ is the size of the chiral molecules expressed in $[\mathrm{nm}]$. Finally, Eq. (1) can be rewritten as:

$$
J(x, t)=\frac{Q_{C M}+Q_{A M} \operatorname{Pr}(A M \rightarrow C M)}{\sqrt{(4 \pi D t)^{3}}} e^{-\frac{x^{2}}{4 D t}}
$$

where $Q_{C M}$ is the initial concentration of chiral molecules, $Q_{A M}$ is the initial concentration of achiral molecules and $J(x, t)$ represents the Brownian particles at time $t$ at point $x$, with first moment as:

$$
x^{2}=2 D t,
$$

and standard deviation:

$$
\sigma=\sqrt{2 D t}
$$

In Eq. (3), we account for the achiral molecules that are "inducted" to become chiral with a certain probability that is proportional to the helical twisting power of the chiral molecules, i.e.:

$$
\operatorname{Pr}(A M \rightarrow C M) \backsim \beta,
$$

where $\beta$ is the helical twisting power and is expressed as [24]

$$
\beta=\frac{\Delta \mu}{8 \pi K_{2} k},
$$

where $\Delta u$ is the chemical potential difference between a chiral molecule and its enantiomer when they are placed in the solution, $K_{2}$ is twist elastic constant, the wavevector $k=2 \pi / P$ and $P$ is the elical pitch, which is inversely proportional to the concentration of chiral molecules injected by the transmitter.

\section{CHIRAL OPTICAL CHANNEL}

In the context of molecular communication, we envision that chiral molecules will be expected to be largely exploited [25]. Due to the feature of changing the polarization plane of an impinging optical signal, data information can be encoded into chiral molecules, and carried out via the chiral transfer mechanism. Specifically, when an optical pulse impinges a (biological) chiral channel, an optical activity as output of the channel will be observed. The optical activity is expressed as a rotation of the polarization plane of the impinging EM wave. On the other side, if no pulse impinges the chiral channel, no optical activity will be observed at the output of the chiral channel, and then, there will be no rotation of the polarization plane of the EM wave.

In Fig. 4 we show how the chiral molecules are arranged after the diffusion process, in a steady state. We assume that a certain concentration of chiral molecules is injected in the system and these molecules diffuse in the solution and "transfer" their chirality to other achiral molecules. Specifically, a Tx nanomachine releases a concentration of chiral molecules that transfer chirality to neighboring achiral molecules, which become chiral as well. Blue circles represent chiral molecules (both enantiomers), while gray circles are the achiral molecules. In this work, we treat chiral molecules as chiral optical antennas and we focus on some specific parameters allowing the characterization of the chiral optical field generated. In particular, as demonstrated in [26], we consider the chirality flux 


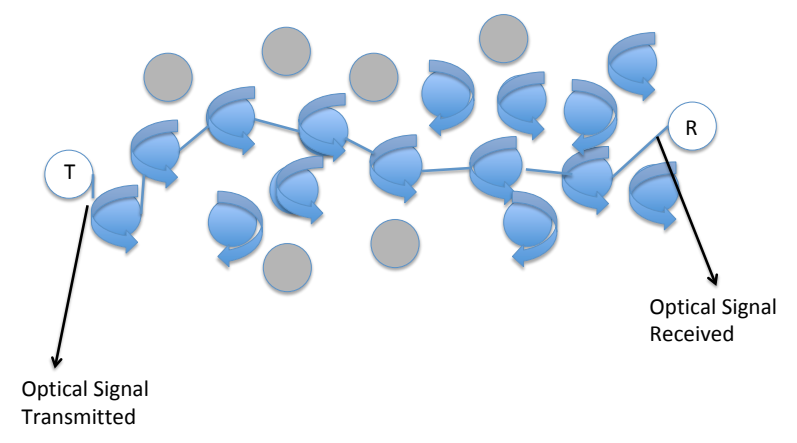

Fig. 4 - Representation of the chiral molecular channel after the diffusion process. The blue (gray) circles represent chiral (achiral) molecules.

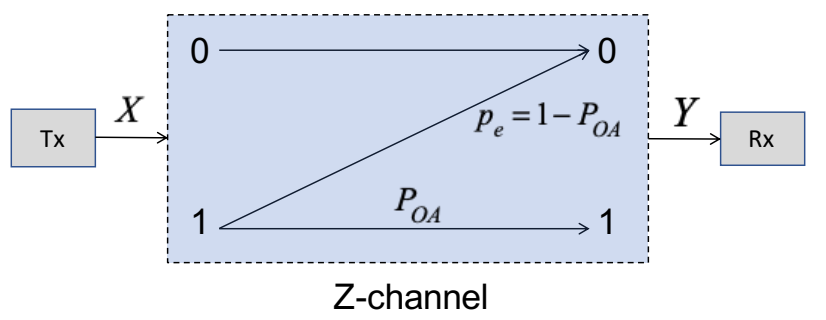

Fig. 5 - Z-channel model for the chiral medium.

efficiency, that describes the ability of our molecules to scatter chiral optical fields, that will be considered as the input for the neighbor chiral molecule as shown in Fig. 4. In particular, the chiral flux efficiency can be written as [26]:

$$
\eta_{\tilde{F}, d}=\operatorname{Re}\left(\alpha_{c}\right) \frac{3}{8 \mu_{0}} \frac{\left|\varepsilon_{0}\right|^{2}}{P_{t o t}} \frac{1}{\sqrt{r^{2}+\zeta^{2}}},
$$

with $r$ and $\zeta$ as radial and longitudinal cylindrical coordinates, $\mu_{0}$ and $\left|\varepsilon_{0}\right|^{2}$ the amplitude of the complex electric field in the point 0 and $P_{t o t}$ is the total power of the outgoing light. Most important is the parameter $\alpha_{c}$ representing the coupled magneto-electric polarizability, to which chiral optical properties are attributed to. Of course, we have to consider that in molecules chiral optical signals are lower than in chiral metallic nanostructures, but there are recent research activities showing how it is possible to improve the quality of the signal [27].

The chiral channel can be then designed as a Z-channel, as depicted in Fig. 5. The output of the channel is expressed in terms of rotation of the polarization plane in case of an optical wave impinging the biological chiral channel.

From the telecommunications point of view, the optical activity due to the effect of chirality can be decoded as a 1 bit, while the absence of rotation of the polarization plane will be decoded as a 0 bit. Fig. 5 describes a chiral channel comprised of chiral molecules. A source node (i.e., Tx node) emits a bit stream modulated through an On Off Keying (OOK) scheme. Specifically, the variable $X$ represents the bit 1 or 0 transmitted along the channel, while $Y$ is the received bit (i.e., 1 or 0 ), based on a probabilistic approach. The presence of a bit 1 at the receiver means that a bit 1 has been transmitted with conditional probability $\operatorname{Pr}(Y=1 \mid X=1)$, while a bit 0 at the receiver side can be affected by errors in the channel corresponding to the error probability $p_{e}=\operatorname{Pr}(Y=0 \mid X=1)$, in case of a bit 1 transmitted with errors. Notice that, different to traditional 00K-based communication schemes for molecular communications, in this paper the 00K modulation is not based on the concentration variation of the molecules, but on the optical activity generated by the chiral molecules. In practice, a bit 1 is associated to the optical activity occurrence, while a bit 0 is associated to no optical activity at the output of the channel.

Furthermore, we assume a time-based synchronization scheme at the transmitter side, and then the transmission of a bit 1 corresponds to an EM wave that excites the chiral channel at the beginning of a time slot, while no excitation corresponds to the emission of a bit 0 . The probability of sending a bit 0 in the case of No Excitation at the beginning of the time slot is defined as $P_{N E}$. If no excitation is provided to the Tx node, then no optical activity will occur (i.e., from the information theory point of view, no transmission errors will occur, while transmitting bit 0 ). The probability that the bit 1 is correctly received by the receiver corresponds to the probability of optical activity experienced by the chiral molecules, namely $P_{O A}$. This probability can be considered depending on the specific optical rotation i.e., $[\alpha]_{\lambda}^{T}$, that is a physical constant of a chiral molecule, expressed as:

$$
[\alpha]_{\lambda}^{T}=\frac{\alpha}{l \cdot \rho},
$$

where $\alpha$ is the optical rotation expressed in degrees, $l$ is the optical path length $[d m]$, and $\rho$ is the concentration of sample in $[\mathrm{g} / \mathrm{mL}]$, that we can derive from ((3)). In Eq. (9), we notice that the specific rotation depends on the wavelength $\lambda[\mathrm{nm}]$ of the impinging EM wave and the temperature $T$ [Celsius]. Usually, the wavelength of the light used is $589 \mathrm{~nm}$ (i.e., the sodium $D$ line), and the symbol $D$ is used i.e., $[\alpha]_{D}^{T}$. The specific rotation can be either positive or negative, if the chiral molecules are dextrotary or levorotary, respectively.

The probability of optical activity occurrence is intrinsically not equal to 1 , i.e., $P_{O A} \neq 1$, and can be expressed as:

$$
P_{O A}=\operatorname{Pr}\left\{[\alpha]_{\lambda}^{T}>+0^{\circ}\right\},
$$

that means that if the specific rotation is greater than $+0^{\circ}$, then it is likely to have optical activity at the output of the chiral channel. Notice that the specific rotation depends on the enantiomers that will rotate the plane of the polarized light of the same magnitude but in opposite directions (i.e., + or - ). Without loss of generality, herein we assumed a positive rotation of the polarization plane, i.e., we are assuming that the biological medium is comprised of a mixture of enantiomers $(+/-)$ but the overall contribution of the specific rotation will be positive. On the 


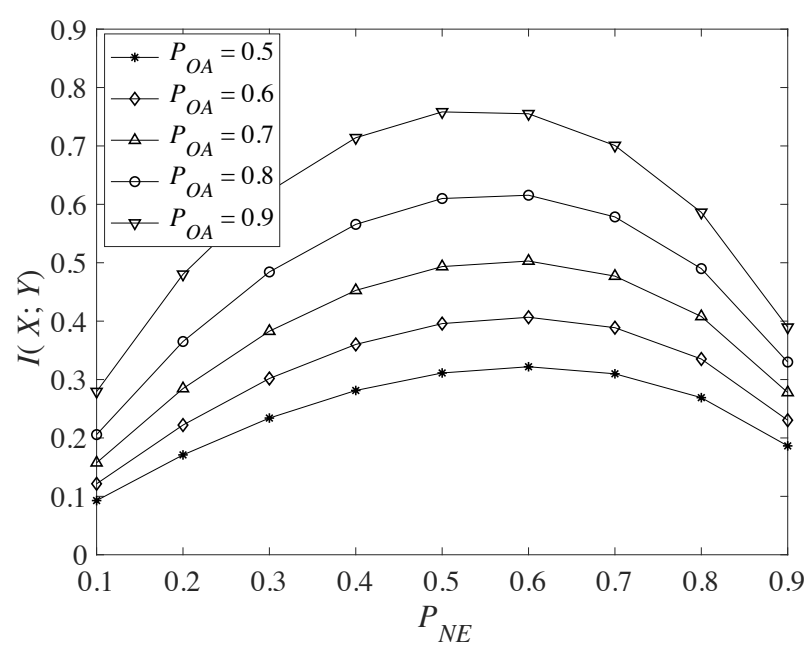

Fig. 6 - Mutual information related to the enantiomer Z-channel model.

other side, the channel will be optically inactive if the specific rotation will be null i.e., there will be both $50 \%(+)$ and $50 \%(-)$ of enantiomers. This configuration is a racemate or racemic mixture.

According to the transmission probabilities of the Zchannel, the associated transition matrix is

$$
\mathbf{P}=\left[\begin{array}{cc}
1 & 0 \\
1-P_{O A} & P_{O A}
\end{array}\right],
$$

and then we can derive the mutual information between $X$ and $Y$ as:

$I(X ; Y)=H\left(P_{O A}\left(1-P_{N E}\right)\right)-\left(1-P_{N E}\right) H\left(1-P_{O A}\right)$,

where $H(\cdot)$ represents the binary entropy. From Eq. (12), we can compute the channel capacity of the chiral medium as the maximum of the mutual information i.e.,

$C_{\text {chiral }}=\max _{P_{O A}}\left[H\left(P_{O A}\left(1-P_{N E}\right)\right)-\left(1-P_{N E}\right) H\left(1-P_{O A}\right)\right]$,

where the probability distribution of input that maximizes the capacity will change according to specific chiral molecule concentrations.

Finally, Fig. 6 depicts the mutual information versus the probability of no excitation, for different values of $P_{O A}$. Notice that we consider only values of $P_{O A}$ higher than 0.5 , as it depends on the concentration of positive/negative enantiomers that comprise the channel. As expected, the highest value of the capacity (i.e., 0.758 bit) is obtained for a high value of $P_{O A}$.

\section{CONCLUSIONS}

This paper presents an overview of the chirality effect in biomolecules. Chiral molecules i.e., enantiomers, are present in nature everywhere, and therefore the main applications range from pharmaceutical to chemical and biological fields, including also communications and electronics. Apart from natural enantiomers, artificial chiral materials (i.e., chiral metamaterials) can be accordingly designed in order to exhibit an enhanced optical activity i.e., the Giant Optical Activity (GOA) effect.

In the context of communications, the use of natural chiral molecules, as well as chiral metamaterials, is envisioned as a potential enabler for novel communication techniques. Specifically, in an MC paradigm, chiral molecules have been analyzed as viable candidates for chiral communications, where information is encoded into chiral molecules. The features of rotation of the polarization plane and the chirality transfer have been exploited in order to derive a communication model based on chiral molecules. Information is represented by the optical activity, which can propagate inside a chiral medium by means of chirality transfer.

\section{REFERENCES}

[1] I. F. Akyildiz, J. M. Jornet, and M. Pierobon. "Nanonetworks: a New Frontier in Communications". In: Communications of the ACM 54 (11 Nov. 2011), pp. 84-89.

[2] Ian F. Akyildiz, Fernando Brunetti, and Cristina Blázquez. "Nanonetworks: A new communication paradigm". In: Computer Networks 52.12 (2008), pp. 2260-2279. ISSN: 1389-1286. DoI: https : / / doi.org/10.1016/j.comnet. 2008.04.001. URL: http : / / www . sciencedirect . com / science / article/pii/S1389128608001151.

[3] M. Pierobon and I. F. Akyildiz. "A physical endto-end model for molecular communication in nanonetworks". In: IEEE Journal on Selected Areas in Communications 28.4 (May 2010), pp. 602-611. ISSN: 0733-8716. DOI: 10 . 1109 / JSAC . 2010 . 100509.

[4] T. Nakano, M. J. Moore, F. Wei, A. V. Vasilakos, and J. Shuai. "Molecular Communication and Networking: Opportunities and Challenges". In: IEEE Transactions on NanoBioscience 11.2 (June 2012), pp. 135-148. ISSN: 1536-1241. DOI: 10.1109/TNB. 2012.2191570.

[5] N. Kim and C. Chae. "Novel Modulation Techniques using Isomers as Messenger Molecules for Nano Communication Networks via Diffusion". In: IEEE Journal on Selected Areas in Communications 31.12 (Dec. 2013), pp. 847-856. ISSN: 0733-8716. DOI: 10.1109/JSAC. 2013. SUP2 . 12130017.

[6] Serena Arnaboldi, Mirko Magni, and Patrizia Romana Mussini. "Enantioselective selectors for chiral electrochemistry and electroanalysis: Stereogenic elements and enantioselection performance". In: Current Opinion in Electrochemistry 8 (2018), pp. 60-72. ISSN: 2451-9103. DOI: https : //doi.org/10.1016/j.coelec. 2018.01.002. URL: http : / / www . sciencedirect . com / science/article/pii/S2451910317301588. 
[7] Michael M. Bobek, Daniel Krois, and Udo H. Brinker. "Induced Circular Dichroism of Cyclodextrin Inclusion Complexes: Examining the Cavity with a Bilateral Probe". In: Organic Letters 2.14 (2000). PMID: 10891214, pp. 1999-2002. DOI: $10.1021 /$ ol005923d.

[8] T. Chen, S.Y. Li, D. Wang, M. Yao, and L.J. Wan. "Remote Chiral Communication in Coadsorber Induced Enantioselective 2D Supramolecular Assembly at a Liquid/Solid Interface". In: Wiley Angewandte Chemie, GDCh (Feb. 2015). DoI: https : / / doi.org/10.1002/anie.201410927.

[9] X. Li and M. Shapiro. "Communications: Spatial separation of enantiomers by coherent optical means". In: The Journal of Chemical Physics 132.4 (2010), p. 041101. DoI: 10.1063/1.3298585.

[10] Ulrich Knof and Alex von Zelewsky. "Predetermined Chirality at Metal Centers". In: Angewandte Chemie International Edition 38.3 (1999), pp. 302322. DoI: 10.1002/(SICI) 1521-3773 (19990201) 38:3<302: :AID-ANIE302>3.0.CO;2-G.

[11] D. Parker, R. S. Dickins, H. Puschmann, C. Crossland, and J. A. K. Howard. "Being excited by lanthanide coordination complexes: aqua species, chirality, excited-state chemistry, and exchange dynamics". In: Chem Rev 102.6 (Feb. 2002), pp. 19772010.

[12] Helen C. Aspinall. "Chiral Lanthanide Complexes: Coordination Chemistry and Applications". In: Chemical Reviews 102.6 (2002). PMID: 12059255, pp. 1807-1850. DOI: 10.1021/cr010288q.

[13] Hiroshi Tsukube and Satoshi Shinoda. "Lanthanide Complexes in Molecular Recognition and Chirality Sensing of Biological Substrates". In: Chem. Rev. 102.6 (June 2002), pp. 2389-2404.

[14] D. Parker. "Excitement in f block: structure, dynamics and function of nine-coordinate chiral lanthanide complexes in aqueous media". In: NCBI, Chem. Soc. Rev. 33.3 (Mar. 2004), pp. 156-165.

[15] J.I. Bruce, D. Parker, S. Lopinski, and R.D. Peacock. "Survey of factors determining the circularly polarised luminescence of macrocyclic lanthanide complexes in solution". In: NCBI 14.7 (July 2002), pp. 562-7.

[16] J.I. Bruce, D. Parker, S. Lopinski, and R.D. Peacock. "Synthesis and characterisation of highly emissive and kinetically stable lanthanide complexes suitable for usage "in cellulo"”. In: NCBI, Org. Biomol. Chem. 3.6 (Mar. 2005), pp. 1013-24.

[17] Jean-Claude G. Benzli. "Benefiting from the Unique Properties of Lanthanide Ions". In: Accounts of Chemical Research 39.1 (2006). PMID: 16411740, pp. 53-61. DoI: 10 . 1021 / ar0400894. eprint: https://doi.org/10.1021/ar0400894.
[18] Sushobhan Ghosh and Partha Sarathi Mukherjee. "Self-Assembly of Molecular Nanoball: Design, Synthesis, and Characterization". In: The Journal of Organic Chemistry 71.22 (2006). PMID: 17064013, pp. 8412-8416. DOI: 10.1021/jo061311g.

[19] Kyung Seok Jeong, Young Shin Kim, Yun Ju Kim, Eunsung Lee, Ji Hye Yoon, Won Hwa Park, Young Woo Park, Seung-Joon Jeon, Zee Hwan Kim, Jaheon Kim, and Nakcheol Jeong. "Lanthanitin: A Chiral Nanoball Encapsulating 18 Lanthanum Ions by Ferritin-Like Assembly". In: Angewandte Chemie International Edition 45.48 (2006), pp. 8134-8138. DOI: 10.1002 /anie. 200603622. eprint: https : // onlinelibrary.wiley.com/doi/pdf/10.1002/ anie. 200603622. URL: https ://onlinelibrary . wiley.com/doi/abs/10.1002/anie. 200603622.

[20] Ben L. Feringa, Richard A. van Delden, Nagatoshi Koumura, and Edzard M. Geertsema. "Chiroptical Molecular Switches”. In: Chemical Reviews 100.5 (2000). PMID: 11777421, pp. 1789-1816. DOI: 10 . $1021 / \operatorname{cr} 9900228$.

[21] Elisa Murguly, Tyler B. Norsten, and Neil R. Branda. "Nondestructive Data Processing Based on Chiroptical 1,2-Dithienylethene Photochromes". In: Angewandte Chemie International Edition 40.9 (2001), pp. 1752-1755. DOI: 10 . 1002 / 1521 3773(20010504) $40: 9<1752:$ : AID-ANIE17520> 3.0. CO;2-W.

[22] Zhaohua Dai, Jennifer Lee, and Wenyao Zhang. "Chiroptical Switches: Applications in Sensing and Catalysis". In: Molecules 17.2 (2012), pp. 1247-1277. ISSN: 1420-3049. DOI: $10.3390 /$ molecules17021247. URL: http : / / www . mdpi . com/1420-3049/17/2/1247.

[23] Ershad Mohammadi, Kosmas Tsakmakidis, AmirNader Askarpour, Parisa Dehkhoda, Ahad Tavakoli, and Hatice Altug. "Nanophotonic Platforms for Enhanced Chiral Sensing". In: ACS Photonics (May 2018). DOI: 10.1021 /acsphotonics . 8b00270.

[24] Mark R. Wilson and David J. Earl. "Calculating the helical twisting power of chiral dopants". In: Journal of Materials Chemistry 11 (2001), pp. 26722677. DOI: $10.1039 / \mathrm{b} 103520 \mathrm{~g}$.

[25] Y. Zhao, A. N. Askarpour, L. Sun, J. Shi, X. Li, and A. Alú. "Chirality detection of enantiomers using twisted optical metamaterials". In: Nature Communications 8 (2017).

[26] Lisa V. Poulikakos, Prachi Thureja, Alexia Stollmann, Eva De Leo, and David J. Norris. "Chiral Light Design and Detection Inspired by Optical Antenna Theory". In: ACS Nano Letters 18 (2018), pp. 46334640. DOI: 10.1021 /acs . nanolett . 8b00083.

[27] VE Bochenkov and TI Shabatina. "Chiral Plasmonic Biosensors”. In: Biosensors (Basel) 8 (4 2018). 


\section{AUTHORS}

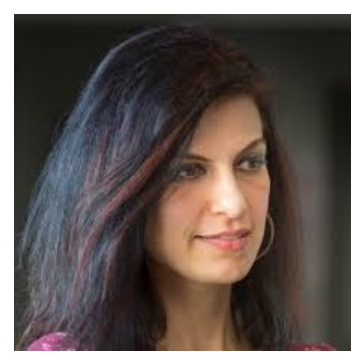

Valeria Loscrí is a permanent researcher of the FUN Team at Inria Lille-Nord Europe since Oct. 2013. From Dec. 2006 to Sept. 2013, she was Research Fellow in the TITAN Lab of the University of Calabria, Italy. She received her $\mathrm{MSc}$ and $\mathrm{PhD}$ degrees in Computer Science in 2003 and 2007, respectively, from the University of Calabria and her HDR (Habilitation a diriger des recherches) in 2018 from Université de Lille (France). Her research interests focus on emerging technologies for new communication paradigms such as Visible Light Communication and TeraHertz bandwidth and cooperation and coexistence of wireless heterogeneous devices. She has been involved in the activity of several European Projects (H2020 CyberSANE, FP7 EU project VITAL, the FP6 EU project MASCOT, etc.), Italian and French projects. She is in the editorial board of IEEE COMST, Elsevier ComNet, JNCA, IEEE Trans. on Nanobioscience. Since 2019, she is Scientific International Delegate for Inria Lille-Nord Europe.

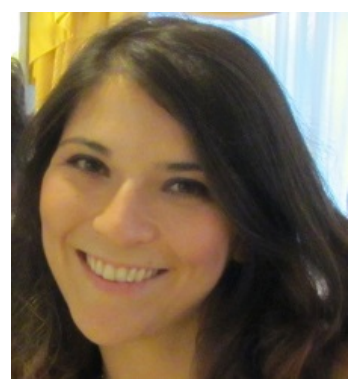

Anna Maria Vegni (Senior member, IEEE) is a tenuretrack Assistant Professor in the Department of Engineering at Roma Tre University (Italy), since March 2020. She received the Ph.D. degree in Biomedical Engineering, Electromagnetics and Telecommunications from the Department of Applied Electronics, Roma Tre University, in March 2010. She received the 1st and 2nd level Laurea Degree cum laude in Electronics Engineering at Roma Tre University, in July 2004, and 2006, respectively. In 2009, she was a visiting researcher in the Multimedia Communication Laboratory, directed by Prof. Thomas D.C. Little, at the Department of Electrical and Computer Engineering, Boston University, Boston, MA. Her research activity focused on vehicular networking supported by heterogeneous wireless networks and optical wireless communications. She a member of ACM and an IEEE Senior Member. In March 2018, she got the Italian Habilitation (Abilitazione Scientifica Nazionale) for Associate Professorship in Telecommunication Engineering. She is involved in the organization of several IEEE and ACM international conferences and is a member of the editorial board of IEEE Communications Magazine, Ad Hoc Networks, Journal of Networks and Computer Applications, Nanocomnet Elsevier journals, IEEE JCN, ITU J-FET and ETT Wiley journal. 